\title{
Classical model for bulk-ensemble NMR quantum computation
}

\author{
Rüdiger Schack* ${ }^{(\mathrm{a}, \mathrm{b})}$ and Carlton M. Caves $^{\dagger}{ }^{\text {(a) }}$ \\ (a) Center for Advanced Studies, Department of Physics and Astronomy, \\ University of New Mexico, Albuquerque, NM 87131-1156, USA \\ (b) Department of Mathematics, Royal Holloway, \\ University of London, Egham, Surrey TW20 0EX, UK
}

(November 13, 2018)

\begin{abstract}
We present a classical model for bulk-ensemble NMR quantum computation: the quantum state of the NMR sample is described by a probability distribution over the orientations of classical tops, and quantum gates are described by classical transition probabilities. All NMR quantum computing experiments performed so far with three quantum bits can be accounted for in this classical model. After a few entangling gates, the classical model suffers an exponential decrease of the measured signal, whereas there is no corresponding decrease in the quantum description. We suggest that for small numbers of quantum bits, the quantum nature of NMR quantum computation lies in the ability to avoid an exponential signal decrease.
\end{abstract}

\section{INTRODUCTION}

The original proposals [1,2] for quantum computing using high-temperature, liquid-state nuclear magnetic resonance (NMR) sparked an explosion of interest. The resulting work on NMR quantum-information processing has attracted a great deal of attention. In addition to a long list of publications [1 20], there have been numerous news reports, especially on the recent NMR experiments on quantum error correction [5] and quantum teleportation 酒.

The fundamental information-processing elements in NMR are two-level nuclear spins, called quantum bits, or qubits for short, which are bound together in a single molecule. A liquid NMR sample contains a macroscopic number of molecules, each of which functions as an independent information-processing system. The molecules are initially in thermal equilibrium at high temperature; the nuclear spins are only weakly polarized along the direction of a strong applied magnetic field. NMR techniques cannot control the quantum states of

*E-mail: r.schack@rhbnc.ac.uk

†E-mail: caves@tangelo.phys.unm.edu 
individual molecules; instead, all the molecules in the sample are manipulated in parallel. Moreover, NMR readout techniques are sensitive to the average magnetization of the entire sample. For these reasons the use of high-temperature, liquid-state NMR techniques for quantum-information processing is called bulk-ensemble quantum computation. Clever techniques have been devised to map the coherent quantum-mechanical manipulations required for quantum computation to this situation in which one neither controls nor makes measurements on individual molecules [1, 2, 8, 8,11,13].

This paper deals exclusively with bulk-ensemble quantum-information processing, not with proposals (see, for example, [21]) for using nuclear spins in situations, as in solid-state systems, where they can be highly polarized. Although the paper is phrased in terms of and aimed at NMR quantum computation, the analysis is not specific to NMR and could be applied to any realization of bulk-ensemble quantum computation.

Bulk-ensemble NMR quantum computation is in principle scalable to many qubits [20], but it is unclear whether it will ever lead to useful quantum computations involving more than a few dozen qubits [1, [3. Nevertheless, the experiments performed so far, which use only a few qubits, are cited as important demonstrations of the principles of quantum-information processing and quantum computation. There has, however, been persistent skepticism about whether these experiments demonstrate genuine quantum-information processing. Recently these doubts have been brought into sharper focus [22] with the realization that all quantum states used in present NMR experiments are separable [23], i.e., unentangled. Entanglement is generally thought to be an essential feature of quantum computation 24 26.

In this paper we explore the "quantumness" of bulk-ensemble quantum computation. There being no general definition of what it means to be doing genuine quantum-information processing, we begin by proposing three different criteria, which are investigated in the remainder of the paper.

- According to the first criterion, an $N$-qubit NMR experiment does not demonstrate genuine quantum-information processing if all quantum states that occur during the experiment are separable (unentangled), i.e., are equivalent to classical ensembles of spinning tops. The motivation for this criterion is that for separable states, the statistics of any measurement performed on the NMR sample can be described in the language of classical probabilities for spin orientations. With respect to this criterion, NMR experiments for up to about 15 qubits do not demonstrate genuine quantum-information processing 23]. There is, however, an immediate objection to this criterion: even if all states involved in the experiment are classical, the transformations between the states might be essentially quantum mechanical, not having any description in classical language.

- Our second criterion addresses this objection by stating that an $N$-qubit NMR experiment does not demonstrate genuine quantum-information processing if we can construct an overall classical model for the experiment, by which we mean that in addition to the experiment's satisfying the first criterion, the transformation $T$ that maps the initial quantum state to the final quantum state can be described by classical transition probabilities. The transition probabilities are required to be fully specified by $T$; in particular, they must be independent of the initial and final states. We formulate an overall classical model below, which shows that, with respect to this 
second criterion, NMR experiments for up to about 6 qubits do not demonstrate genuine quantum-information processing. An objection to this second criterion is that an overall classical model does not constitute a valid model for a quantum computation, because the complexity of the overall transition probabilities might increase exponentially with problem size; according to this objection, computational complexity is at the heart of the question of whether a given computation is quantum mechanical.

- Our third criterion incorporates this objection as follows. It states that an $N$-qubit NMR experiment does not demonstrate genuine quantum information processing if we can construct a gate-by-gate classical model, by which we mean that in addition to the first criterion being satisfied, any gate $U$ implemented in the experiment can be described by classical transition probabilities, which, as before, are fully specified by $U$ and which, apart from trivial contributions for the qubits not involved in the gate, are independent of the total number of qubits [27]. We devise a gate-by-gate classical model below. It shows that all 3-qubit quantum computing experiments performed so far 48 do not demonstrate genuine quantum-information processing according to this third criterion.

In the NMR literature, transformations are often represented by diagrams that depict spins as arrows evolving on spheres; see, e.g., [28] or the "effective fields picture" in [14, where a diagram representing a controlled-NOT gate is given. This diagram illustrates what is new in our approach. The diagram assumes that the sample is split into two subpopulations, according to whether the control qubit is up or down. Thus it provides a classical description of the gate for initial conditions corresponding to computational basis states of the control qubit, but not for arbitrary, possibly entangled initial states. Consequently, it does not give a classical model of the controlled-NOT gate in the sense of our third criterion.

We emphasize that our gate-by-gate classical model is not the same as a simulation of a quantum algorithm on a classical computer. First, in our classical model, measurement statistics are determined by probabilities for classical spin orientations; in a classical simulation, where one calculates the quantum amplitudes in, say, the spin-up-spin-down, or computational basis, one cannot go from the amplitudes to measurement statistics using classical probability logic. This means that our classical model provides a hidden-variable description of the experiment [27], but a classical simulation does not. Second, the computational complexity of a classical simulation increases exponentially with the number of gates [24], whereas the complexity of our transition probabilities for an entangling gate increases only linearly with the number of qubits.

Equally important is a difference between our gate-by-gate classical model and the quantum description of an NMR experiment. In our classical model, the strength of the magnetization signal decreases exponentially with the number of entangling gates. Though this decrease can be staved off for a few entangling steps, it cannot be put off indefinitely. Such a decrease is absent from the quantum description. Thus it is straightforward to design experiments that cannot be described by our classical model. Two-qubit experiments that fall into this class are [9] and probably also [10], although in the latter case this is not clear from the published data. In order to prove conclusively, however, that a bulk-ensemble NMR experiment demonstrates genuine quantum-information processing in the sense of the 
third criterion given above, one must rule out all gate-by-gate classical models, not just the particular model given in this paper.

The paper is organized as follows. In Sec. [1], we give a brief description of bulk-ensemble NMR quantum computation, and in Sec. III, we summarize the proof given in [23] that the states used in high-temperature NMR experiments are classical up to about 15 qubits in the sense of the first criterion above. In Sec. IV, we define the transition probabilities that are used in our overall and gate-by-gate classical models. Used directly in a gate-by-gate classical model, these transition probabilities give rise to a signal decrease by a constant factor at each entangling multi-qubit operation. Section $\nabla$ shows how to modify the gate-by-gate model so as to avoid this signal decrease for a small number of entangling steps. In Sec. VI we consider the implications of our results for bulk-ensemble NMR quantum-information processing.

\section{NMR QUANTUM-INFORMATION PROCESSING}

All NMR quantum computing experiments performed so far [16] work according to the following principles. The state of the sample is described by a density operator $\rho$ for the $N$ spins (qubits) in each molecule. The molecules are prepared in an initial state

$$
\rho=(1-\epsilon) M+\epsilon \rho_{1},
$$

where $M=I / 2^{N}$ is the maximally mixed density operator for $N$ qubits ( $I$ is the unit operator) and $\rho_{1}$ is a density operator, usually chosen to be the projector onto the computational basis state $|0 \ldots 0\rangle$. The parameter $\epsilon$ scales like

$$
\epsilon=\frac{\alpha N}{2^{N}} \text {. }
$$

Here $\alpha=h \bar{\nu} / 2 k T$, where $\bar{\nu}$ is the average resonant frequency of the active spins in the strong magnetic field, determines the polarization of the sample. If $\rho_{1}$ is a pure state, $\rho$ is called a pseudopure state [自]. Procedures for synthesizing a pseudopure state from an initial thermal density operator are described in [2,8, 11, 13,

For typical molecules in present magnetic-field strengths, the average resonant frequency is roughly $\bar{\nu} \sim 300 \mathrm{MHz}$, which at room temperature, gives $\alpha \sim 2 \times 10^{-5}$. It is difficult to know the actual value of $\alpha$ and $\epsilon$ in the experiments, because the polarization is not absolutely calibrated. In evaluating the experiments, we adopt the scaling of Eq. (2.2), with a conservative value of $\alpha=2 \times 10^{-6}$, which is meant to take into account inefficiencies in the experiments, especially loss of polarization involved in the synthesis of a pseudopure state.

One interpretation of $\epsilon$ is that it specifies the fraction of molecules in the sample that occupy the desired initial state $\rho_{1}$. This interpretation is not unique, and it is not mandated or even preferred by quantum mechanics. It is typically promoted to the level of a physical fact by advocates of NMR quantum computation, yet it becomes a physical fact only if one actually prepares a fraction of the molecules in a particular state [29], a situation that does not apply to a high-temperature NMR experiment. The freedom to interpret $\rho$ in terms of other ensembles underlies the conclusions of this paper.

After synthesis of the desired initial state, a sequence of radio-frequency pulses, alternating with continuous evolution, is applied to the sample. We first consider the case where 
the evolution is described by a unitary transformation $U$. Applying the unitary operator $U$ to $\rho$ results in the state

$$
\rho^{\text {out }} \equiv U \rho U^{\dagger}=(1-\epsilon) M+\epsilon U \rho_{1} U^{\dagger} \equiv(1-\epsilon) M+\epsilon \rho_{1}^{\text {out }} .
$$

The totally mixed part of the state is unaffected by the unitary transformation. The output state retains the form (2.1) with the same value of $\epsilon$, but - and this is the essence of the bulk-ensemble paradigm for quantum computation - $\rho_{1}$ undergoes the desired unitary transformation.

The same conclusions hold for transformations implemented using gradient pulses together with diffusion in the sample [8]; these are equivalent to mixtures of unitary transformations, as in

$$
\rho^{\text {out }} \equiv \sum_{l} p_{l} U_{l} \rho U_{l}^{\dagger}=(1-\epsilon) M+\epsilon \sum_{l} p_{l} U_{l} \rho_{1} U_{l}^{\dagger} \equiv(1-\epsilon) M+\epsilon \rho_{1}^{\text {out }}
$$

where the $p_{l} \geq 0$ are probabilities. We assume that decoherence also preserves the form of the density operator, leaving $\epsilon$ unchanged. This assumption is certainly justified when decoherence is simulated by unitary transformations, as in the gradient pulses used to simulate decoherence in [5]. It is also true for naturally occurring decoherence for times short compared to the characteristic time for relaxation to thermal equilibrium. These are the times of interest for quantum-information processing.

The last step in any NMR experiment is the readout. By applying radio-frequency pulses and then measuring the transverse magnetization of the sample, one can determine all expectation values of the form

$$
\operatorname{tr}\left(\rho^{\text {out }} \sigma_{\beta_{1}} \otimes \cdots \otimes \sigma_{\beta_{N}}\right)=\epsilon \operatorname{tr}\left(\rho_{1}^{\text {out }} \sigma_{\beta_{1}} \otimes \cdots \otimes \sigma_{\beta_{N}}\right) .
$$

The tensor product in this expression includes one operator for each spin; $\sigma_{\beta_{k}}$ denotes the unit operator $I$ for the $k$ th spin if $\beta_{k}=0$, and it denotes a Pauli matrix if $\beta_{k}=1,2$, or 3 . In writing Eq. (2.5) and all such expectation values in this paper, it is assumed that there is at least one Pauli matrix in the tensor product (not all the $\beta$ 's are zero). The maximally mixed part of the density operator does not contribute to the measured expectation values, which are determined by the state $\rho_{1}^{\text {out }}$ that undergoes the desired evolution. The parameter $\epsilon$ appears naturally as a measure of the strength of the magnetization signal (or of the signal-to-noise ratio).

\section{SEPARABILITY OF STATES USED IN NMR}

Our conclusions rest on the freedom to write states of the form (2.1) in terms of probability distributions over spin orientations for $N$ classical tops. We review one such representation [23], the foundation for our work, which provides a classical description for all states of the form (2.1) provided that

$$
\epsilon \leq \eta \equiv \frac{1}{1+2^{2 N-1}}
$$

If we assume that $\alpha=2 \times 10^{-6}$ in Eq. (2.2), this inequality holds for $N<16$ qubits. 
The argument is straightforward [23]. The most general pure product state of $N$ qubits has the form

$$
P_{1}(\tilde{n}) \equiv P_{1}\left(\vec{n}_{1}, \ldots, \vec{n}_{N}\right)=\frac{1}{2^{N}}\left(I+\vec{n}_{1} \cdot \vec{\sigma}\right) \otimes \cdots \otimes\left(I+\vec{n}_{N} \cdot \vec{\sigma}\right)
$$

This state can be interpreted as $N$ classical tops pointing in the directions given by the unit vectors $\vec{n}_{1}, \ldots, \vec{n}_{N}$, denoted collectively by $\tilde{n}$. Since the operators $P_{1}(\tilde{n})$ form an overcomplete basis in the space of linear operators acting on $N$ qubits, any $N$-qubit density operator $\rho$ can be expanded as

$$
\rho=\int d \Omega_{\tilde{n}} w^{\rho}(\tilde{n}) P_{1}(\tilde{n})
$$

where $d \Omega_{\tilde{n}} \equiv d \Omega_{\vec{n}_{1}} \cdots d \Omega_{\vec{n}_{N}}$. The overcompleteness means that the expansion coefficients are not unique. One choice is 23]

$$
w^{\rho}(\tilde{n}) \equiv \operatorname{tr}\left(\rho Q_{1}(\tilde{n})\right)
$$

where the operators $Q_{1}(\tilde{n})$ are defined by

$$
Q_{1}(\tilde{n}) \equiv \frac{1}{(4 \pi)^{N}}\left(I+3 \vec{n}_{1} \cdot \vec{\sigma}\right) \otimes \cdots \otimes\left(I+3 \vec{n}_{N} \cdot \vec{\sigma}\right)
$$

The expansion coefficients $w^{\rho}(\tilde{n})$ can be positive or negative, but they obey the bound

$$
w^{\rho}(\tilde{n}) \geq\left(\begin{array}{c}
\text { smallest } \\
\text { eigenvalue } \\
\text { of } Q_{1}(\tilde{n})
\end{array}\right)=-\frac{2^{2 N-1}}{(4 \pi)^{N}} .
$$

When $w^{\rho}(\tilde{n})$ is everywhere nonnegative, it can be interpreted as a classical probability distribution for the $N$ spins to point in the directions $\vec{n}_{1}, \ldots, \vec{n}_{N}$. Since the maximally mixed density operator $M$ has probability density $w^{M}(\tilde{n})=1 /(4 \pi)^{N}$, it follows that for a density operator $\rho$ of the form (2.1) with $\epsilon \leq \eta$,

$$
w^{\rho}(\tilde{n})=\frac{1-\epsilon}{(4 \pi)^{N}}+\epsilon w^{\rho_{1}}(\tilde{n}) \geq \frac{1-\epsilon / \eta}{(4 \pi)^{N}} \geq 0 .
$$

A density operator for a joint system is separable if it can be written as a nonnegative linear combination of product density operators. When $w^{\rho}(\tilde{n})$ is everywhere nonnegative, the expansion (3.3) provides a separable representation for $\rho$. Related work on the separability of states near the maximally mixed state can be found in [29] and [30]. Separable states have no quantum entanglement [31]. The importance of separability in this paper is that a separable state of $N$ qubits can be interpreted in terms of an ensemble of classical tops, because the expectation values (2.5) have the standard form for an ensemble with probability distribution $w^{\rho}(\tilde{n})$ :

$$
\operatorname{tr}\left(\rho \sigma_{\beta_{1}} \otimes \cdots \otimes \sigma_{\beta_{N}}\right)=\int d \Omega_{\tilde{n}} w^{\rho}(\tilde{n}) \underbrace{\operatorname{tr}\left(P_{1}(\tilde{n}) \sigma_{\beta_{1}} \otimes \cdots \otimes \sigma_{\beta_{N}}\right)}_{=\left(n_{1}\right)_{\beta_{1}} \cdots\left(n_{N}\right)_{\beta_{N}}} .
$$


In this expression, $\left(n_{j}\right)_{\beta_{j}}=1$ if $\beta_{j}=0$, and $\left(n_{j}\right)_{\beta_{j}}$ is a Cartesian component of the vector $\vec{n}_{j}$ if $\beta_{j}=1,2$, or 3 .

If one adopts our first criterion, none of the NMR experiments performed to date has done any genuine quantum-information processing, since they all use 2 or 3 qubits. Yet the fact that all states - initial, intermediate, and final - that occur in a given NMR experiment are equivalent to ensembles of classical tops does not mean, by itself, that there is a classical model for the entire experiment. To see this, consider the following naïve attempt to describe a unitary transformation $U$ by classical transition probabilities:

$$
w^{U \rho U^{\dagger}}(\tilde{n})=\operatorname{tr}\left(U \rho U^{\dagger} Q_{1}(\tilde{n})\right)=\int d \Omega_{\tilde{m}} w^{\rho}(\tilde{m}) \underbrace{\operatorname{tr}\left(U P_{1}(\tilde{m}) U^{\dagger} Q_{1}(\tilde{n})\right)}_{\equiv t_{U}(\tilde{n} \mid \tilde{m})} .
$$

The transition function $t_{U}(\tilde{n} \mid \tilde{m})$ is not a transition probability because it assumes negative values; for example, for the trivial case of the identity transformation, $U=I$, we have

$$
t_{I}(\tilde{n} \mid \tilde{m})=\operatorname{tr}\left(P_{1}(\tilde{m}) Q_{1}(\tilde{n})\right)=\frac{1}{(4 \pi)^{N}} \prod_{j=1}^{N}\left(1+3 \vec{m}_{j} \cdot \vec{n}_{j}\right) .
$$

The first criterion is the right one, however, for judging claims of having produced entangled states using bulk-ensemble NMR. For example, as pointed out in 23, the claim of Laflamme et al. 6] to have created a 3-qubit Greenberger-Horne-Zeilinger (GHZ) entangled state is incorrect. Despite the authors' assertion, "We describe the creation of a GreenbergerHorne-Zeilinger state of the form $(|000\rangle+|111\rangle) / \sqrt{2}$ (three maximally entangled quantum bits) using nuclear magnetic resonance. ... We have thus extended the space of entangled quantum states explored systematically to three quantum bits ...," no entanglement was created in that experiment; the statistics of any measurement performed on the purported GHZ state could have been explained in terms of classical correlations contained in $w^{\rho}(\tilde{n})$. Similar conclusions apply to the experiments in which Chuang et al. [11] and Cory et al. [8] claim to have created 2-qubit Einstein-Podolsky-Rosen (EPR) entangled states. The states created in these experiment were unentangled, though Chuang et al. write, "As an application of the controlled-NOT gate, we used it in a simple quantum circuit to create entangled states from the thermal mixture. ... We have experimentally confirmed this (nonclassical) behavior, and the signature of the entanglement - a purely non-classical effect - is the strong reverse diagonal measured in the density matrix," and Cory et al. refer to applying the "spin-coherence XOR gate to a one-spin superposition to create an entangled state."

Indeed, the first criterion can be applied to any NMR experiment that claims to have manipulated quantum states in a particular way. For example, in describing the NMR version of quantum teleportation [4], a 3-qubit experiment, the authors assert, "Quantum-mechanical systems have information processing capabilities that are not possible with classical devices. One example is quantum teleportation, in which the quantum state of a system is transported from one location to another without moving through the intervening space. ... Here we report an experimental implementation of full quantum teleportation over inter-atomic distances using liquid-state nuclear magnetic resonance." This claim cannot be supported, because the quantum state at all stages of the experiment could be interpreted in terms of classical correlations among spin directions. What the experiment achieved was a reshuffling of these classical correlations. Nonetheless, as noted above, this conclusion does not imply that there is a classical model for the entire experiment. 


\section{TRANSITION PROBABILITIES FOR QUANTUM GATES}

The essence of a classical model lies in the construction of nonnegative transition probabilities to describe unitary transformations. We first consider product unitaries, i.e., unitary transformations of the form $V=V_{1} \otimes \cdots \otimes V_{N}$, and then tackle the tougher task of entangling unitaries, i.e., unitary transformations that are not product unitaries.

The expansion coefficients for the output state are given by

$$
w^{V \rho V^{\dagger}}(\tilde{n})=\operatorname{tr}\left(V \rho V^{\dagger} Q_{1}(\tilde{n})\right)=\operatorname{tr}\left(\rho V^{\dagger} Q_{1}(\tilde{n}) V\right) .
$$

For a product unitary $V$, the unitary transform of $Q_{1}(\tilde{n})$ factors into a product of transformations for each qubit. If we introduce the 3-dimensional rotation operator $R_{k}$ (an orthogonal transformation) corresponding to the unitary operator $V_{k}$, i.e., $V_{k}^{\dagger} \vec{\sigma} V_{k}=R_{k} \vec{\sigma}$, and use the fact that $\vec{n}_{k} \cdot R_{k} \vec{\sigma}=R_{k}^{-1} \vec{n}_{k} \cdot \vec{\sigma}$, then the unitary transform of $Q_{1}(\tilde{n})$ assumes the form

$$
V^{\dagger} Q_{1}(\tilde{n}) V=Q_{1}\left(R_{1}^{-1} \vec{n}_{1}, \ldots, R_{N}^{-1} \vec{n}_{N}\right)=Q_{1}\left(\tilde{R}^{-1} \tilde{n}\right)
$$

Here $\tilde{R}$ stands for the rotations on all the qubits. Now the output expansion coefficients assume the form

$$
w^{V \rho V^{\dagger}}(\tilde{n})=w^{\rho}\left(\tilde{R}^{-1} \tilde{n}\right)=w^{\rho}\left(R_{1}^{-1} \vec{n}_{1}, \ldots, R_{N}^{-1} \vec{n}_{N}\right)
$$

Not surprisingly, the action of the product unitary $V$ is equivalent to rotating each of the classical tops. The corresponding nonnegative transition probabilities are $w(\tilde{n} \mid \tilde{m})=$ $\delta\left(\tilde{R}^{-1} \tilde{n}-\tilde{m}\right)$. This result shows that, for product unitaries, our transition probabilities are equivalent to the simple classical diagrams often used in the NMR literature [28].

Before proceeding to entangling unitaries, we describe how decoherence is incorporated into our classical models. In some experiments decoherence is simulated as a mixture of product unitary transformations; an example is the use of gradient pulses in [5]. Naturally occurring decoherence processes act independently on the various qubits; on time scales short compared to the thermal relaxation time, they preserve the maximally mixed density operator. Any decoherence process that satisfies these two properties can be described as a mixture of product unitaries [32]. Thus decoherence can be handled in our classical models as a mixture of rotations of the qubits.

To describe general, entangling unitaries, we need to introduce some additional notation. For an arbitrary density operator $\rho$ and for $0<\theta \leq 1$, we define

$$
\rho_{\theta} \equiv(1-\theta) M+\theta \rho \text {. }
$$

Furthermore, we define the states

$$
P_{\theta}(\tilde{n}) \equiv(1-\theta) M+\theta P_{1}(\tilde{n})
$$

and associated operators

$$
Q_{\theta}(\tilde{n}) \equiv \frac{1-\theta^{-1}}{(4 \pi)^{N}} I+\theta^{-1} Q_{1}(\tilde{n})
$$


For a general unitary operator $U$, we define the transition probabilities

$$
w_{U}(\tilde{n} \mid \tilde{m}) \equiv \operatorname{tr}\left(U P_{\eta}(\tilde{m}) U^{\dagger} Q_{1}(\tilde{n})\right)=\frac{1-\eta}{(4 \pi)^{N}}+\eta t_{U}(\tilde{n} \mid \tilde{m}) \geq 0
$$

where $t_{U}(\tilde{n} \mid \tilde{m})$ is defined in Eq. (3.9). The nonnegativity of these transition probabilities follows from the argument leading to Eq. (3.7), since $U P_{\eta}(\tilde{m}) U^{\dagger}$ is a state of the form (2.1) with $\epsilon=\eta$.

It is straightforward to write down the transition probabilities for elementary entangling gates. As an illustration, we compute them for the controlled-phase gate,

$$
\begin{aligned}
U=C_{i j} & =|0\rangle\langle 0|\otimes 1+| 1\rangle\langle 1| \otimes \sigma_{3} \\
& =\frac{1}{2}\left(1+\sigma_{3}\right) \otimes 1+\frac{1}{2}\left(1-\sigma_{3}\right) \otimes \sigma_{3},
\end{aligned}
$$

acting on qubits $i$ and $j$. We obtain

$$
\begin{aligned}
w_{C_{i j}}(\tilde{n} \mid \tilde{m})= & \operatorname{tr}\left(C_{i j} P_{\eta}(\tilde{m}) C_{i j} Q_{1}(\tilde{n})\right) \\
= & \frac{1}{(4 \pi)^{N}}\left(1-\eta+\eta \prod_{l \neq i, j}\left(1+3 \vec{m}_{l} \cdot \vec{n}_{l}\right)\right. \\
& \quad \times\left(\left[1+3\left(m_{i}\right)_{3}\left(n_{i}\right)_{3}\right]\left[1+3\left(m_{j}\right)_{3}\left(n_{j}\right)_{3}\right]+9\left(\vec{m}_{i} \times \vec{n}_{i}\right)_{3}\left(\vec{m}_{j} \times \vec{n}_{j}\right)_{3}\right. \\
& \left.\left.\quad+3\left[\left(m_{i}\right)_{3}+3\left(n_{i}\right)_{3}\right]\left(\vec{m}_{j \perp} \cdot \vec{n}_{j \perp}\right)+3\left[\left(m_{j}\right)_{3}+3\left(n_{j}\right)_{3}\right]\left(\vec{m}_{i \perp} \cdot \vec{n}_{i \perp}\right)\right)\right),
\end{aligned}
$$

where $\vec{m}_{\perp}$ is the projection of $\vec{m}$ into the $1-2$ plane, obtained by setting $m_{3}$ to zero. As in this example, the qubits not involved in an entangling gate do appear in the transition probabilities, but in a simple, universal way.

Applying the transition probabilities (4.7) to an input ensemble $w_{\text {in }} \equiv w^{\rho}(\tilde{n})$, we obtain a classical output ensemble

$$
\begin{aligned}
w_{\text {out }}(\tilde{n}) & \equiv \int d \Omega_{\tilde{m}} w_{U}(\tilde{n} \mid \tilde{m}) w_{\text {in }}(\tilde{m}) \\
& =\operatorname{tr}(U \underbrace{\left[\int d \Omega_{\tilde{m}} w^{\rho}(\tilde{m}) P_{\eta}(\tilde{m})\right]}_{=\rho_{\eta}} U^{\dagger} Q_{1}(\tilde{n})) \\
& =w^{U \rho_{\eta} U^{\dagger}}(\tilde{n}) \\
& =\frac{1-\eta}{(4 \pi)^{N}}+\eta w^{U \rho U^{\dagger}}(\tilde{n}) .
\end{aligned}
$$

Unlike the quantum output ensemble $w^{U \rho U^{\dagger}}(\tilde{n})$, the classical output ensemble suffers an increase in the fraction of molecules that are maximally mixed. This means that the magnetization signal produced by the classical output ensemble is a factor of $\eta$ smaller than in the quantum description:

$$
\begin{aligned}
\int d \Omega_{\tilde{n}} w_{\text {out }}(\tilde{n})\left(n_{1}\right)_{\beta_{1}} \cdots\left(n_{N}\right)_{\beta_{N}} & =\operatorname{tr}\left(U \rho_{\eta} U^{\dagger} \sigma_{\beta_{1}} \otimes \cdots \otimes \sigma_{\beta_{N}}\right) \\
& =\eta \operatorname{tr}\left(U \rho U^{\dagger} \sigma_{\beta_{1}} \otimes \cdots \otimes \sigma_{\beta_{N}}\right) .
\end{aligned}
$$


If we use the transition probabilities (4.7) to describe transitions of the spin directions in a gate-by-gate classical model of an NMR quantum computation, the magnetization signal of the model loses a factor of $\eta$ at each entangling gate. Though such a model gives a satisfactory account of an NMR experiment in which one ignores the strength of the signal, we can formulate a better model that avoids the decay for a few entangling gates.

\section{GATE-BY-GATE CLASSICAL MODEL}

The key idea in constructing an improved model is to introduce auxiliary, "hidden" spins $\vec{a}_{1}, \ldots, \vec{a}_{N} \equiv \tilde{a}$, one for each qubit. We also need a "counter index" $k$, which increments by 1 at each entangling gate. In the improved model, we can avoid the decay of the signal for $K$ entangling gates, where $K$ is the largest integer such that

$$
\epsilon \leq \eta^{K+1} .
$$

We assume that $\epsilon \leq \eta^{2}$ to ensure that $K \geq 1$. It is useful to introduce the function

$$
\eta_{k} \equiv \begin{cases}\eta^{K-k}, & 0 \leq k<K, \\ 1, & k \geq K .\end{cases}
$$

Between the $k$ th and $(k+1)$ th entangling gates $(k \geq 0)$, we represent a density operator $\rho$ by the expansion coefficients

$$
w_{k}^{\rho}(\tilde{a}) \equiv \operatorname{tr}\left(\rho Q_{\eta_{k}}(\tilde{a})\right) .
$$

It is easy to show that these expansion coefficients are related to the original coefficients (3.4) by

$$
w_{k}^{\rho}(\tilde{a})=\frac{1-\eta_{k}^{-1}}{(4 \pi)^{N}}+\eta_{k}^{-1} w^{\rho}(\tilde{a})
$$

and that $\rho$ can be expanded as

$$
\rho=\int d \Omega_{\tilde{a}} w_{k}^{\rho}(\tilde{a}) P_{\eta_{k}}(\tilde{a}) .
$$

For density operators of the form (2.1), Eqs. (5.4) and (3.7) imply that

$$
w_{k}^{\rho}(\tilde{a}) \geq \frac{1-\epsilon / \eta^{K+1}}{(4 \pi)^{N}} \geq 0
$$

for all $k \geq 0$. Throughout the experiment, the expansion coefficients $w_{k}^{\rho}(\tilde{a})$ can be interpreted as probability distributions.

In our improved classical model, the gate operations manipulate the hidden spins; the effect of a gate shows up in the observable spins $\tilde{n}$ through the correlations of $\tilde{n}$ with $\tilde{a}$. Between the $k$ th and $(k+1)$ th entangling gates, this correlation is described by the joint distribution

$$
w_{k}^{\rho}(\tilde{n}, \tilde{a}) \equiv q_{k}(\tilde{n} \mid \tilde{a}) w_{k}^{\rho}(\tilde{a}),
$$


where

$$
q_{k}(\tilde{n} \mid \tilde{a}) \equiv \frac{1-\eta_{k}}{(4 \pi)^{N}}+\eta_{k} \delta(\tilde{n}-\tilde{a})
$$

is a conditional probability distribution. The marginal distribution for the observable spin variables,

$$
\int d \Omega_{\tilde{a}} w_{k}^{\rho}(\tilde{n}, \tilde{a})=\frac{1-\eta_{k}}{(4 \pi)^{N}}+\eta_{k} w_{k}^{\rho}(\tilde{n})=w^{\rho}(\tilde{n})
$$

is just the right mixture of $w_{k}^{\rho}(\tilde{n})$ with the uniform distribution to produce the distribution $w^{\rho}(\tilde{n})$ that gives the measured expectation values.

We now define transition probabilities for quantum gates, distinguishing as before between product unitaries and entangling unitaries. For a product unitary $V$, we find, by an argument analogous to the previous one, that

$$
w_{k}^{V \rho V^{\dagger}}(\tilde{n}, \tilde{a})=w_{k}^{\rho}\left(\tilde{n}, \tilde{R}^{-1} \tilde{a}\right) .
$$

The effect of $V$ is to rotate the hidden spins. Decoherence is handled, as previously, by mixtures of product unitaries.

To deal with an entangling unitary transformation $U$, we first note that the transition probabilities $w_{U}(\tilde{a} \mid \tilde{b})$ defined in Eq. (4.7) can be expressed as

$$
w_{U}(\tilde{a} \mid \tilde{b})=\operatorname{tr}\left(U P_{\eta \theta}(\tilde{b}) U^{\dagger} Q_{\theta}(\tilde{a})\right) \geq 0
$$

Using this result, together with Eq. (5.5), we find

$$
\begin{gathered}
\int d \Omega_{\tilde{b}} w_{U}(\tilde{a} \mid \tilde{b}) w_{k}^{\rho}(\tilde{b})=\operatorname{tr}(\underbrace{\left.U \int d \Omega_{\tilde{b}} P_{\eta \eta_{k+1}}(\tilde{b}) w_{k}^{\rho}(\tilde{b})\right]} U^{\dagger,} \quad \begin{array}{ll}
\rho & 0 \leq k<K \\
\rho_{\eta}, & k \geq K
\end{array} Q_{\eta_{k+1}}(\tilde{a})) \\
= \begin{cases}w_{k+1}^{U \rho U^{\dagger}}(\tilde{a}), & 0 \leq k<K, \\
w_{k+1}^{U \rho_{\eta} U^{\dagger}}(\tilde{a}), & k \geq K .\end{cases}
\end{gathered}
$$

To describe the effect of the $(k+1)$ th entangling gate, we use transition probabilities

$$
w_{k+1}^{U}(\tilde{n}, \tilde{a} \mid \tilde{m}, \tilde{b}) \equiv q_{k+1}(\tilde{n} \mid \tilde{a}) w_{U}(\tilde{a} \mid \tilde{b})
$$

which take the input ensemble $w_{\text {in }} \equiv w_{k}^{\rho}(\tilde{n}, \tilde{a})$ to an output ensemble

$$
\begin{aligned}
w_{\text {out }}(\tilde{n}, \tilde{a}) & =\int d \Omega_{\tilde{m}} d \Omega_{\tilde{b}} w_{k+1}^{U}(\tilde{n}, \tilde{a} \mid \tilde{m}, \tilde{b}) w_{\text {in }}(\tilde{m}, \tilde{b}) \\
& =q_{k+1}(\tilde{n} \mid \tilde{a}) \int d \Omega_{\tilde{b}} w_{U}(\tilde{a} \mid \tilde{b}) w_{k}^{\rho}(\tilde{b}) \\
& = \begin{cases}w_{k+1}^{U \rho U^{\dagger}}(\tilde{n}, \tilde{a}), & 0 \leq k<K \\
w_{k+1}^{U \rho_{\eta} U^{\dagger}}(\tilde{n}, \tilde{a}), & k \geq K .\end{cases}
\end{aligned}
$$


We have now constructed a gate-by-gate classical model in which there is no loss of magnetization signal for the first $K$ entangling gates. How this works can be summarized as follows. The initial distribution $w_{0}^{\rho}(\tilde{a})$ of the hidden spin variables is chosen to have as little contribution from the uniform distribution as is consistent with nonnegativity. At each entangling gate, the ensemble of hidden spins suffers an increase in the proportion of molecules that are maximally mixed, but the observable spins retain the statistics of the quantum description by becoming more tightly correlated with the hidden spins. After $K$ entangling gates, the observable and hidden spins become $\delta$-correlated; thus from the $(K+1)$ th entangling gate on, the signal decreases by a factor of $\eta$ at each entangling gate.

\section{DISCUSSION}

Consider a bulk-ensemble NMR experiment whose parameter $\epsilon$ satisfies the condition $\epsilon \leq \eta^{2}$ that underlies the argument in Sec. $\nabla$. If we assume $\alpha=2 \times 10^{-6}$ in Eq. (2.2), this condition is fulfilled for $N \leq 6$ qubits. The model constructed in Sec. $\mathrm{V}$ provides an overall classical model in the sense of our second criterion: the unitary transformation $U$ that maps the initial state to the final state is described in the model, with no loss of magnetization signal, by the transition probabilities $w_{1}^{U}(\tilde{n}, \tilde{a} \mid \tilde{m}, \tilde{b})$; mixtures of such transition probabilities are used to incorporate decoherence. The predictions of the model for the signal derived from the output state are identical to the quantum predictions. Since all NMR experiments to date involve 2 or 3 qubits, this overall classical model applies to all such experiments.

We turn now to the implications of the gate-by-gate classical model constructed in Sec. $\mathrm{V}$. If we assume again that $\alpha=2 \times 10^{-6}$, in a 2-qubit experiment the model proceeds through $K=5$ entangling gates with no signal loss, and in a 3-qubit experiment, through $K=3$ entangling gates. We illustrate the implications by considering a particular 3-qubit experiment, the NMR version of quantum teleportation [1. After preparation of a pseudopure state by the gradient-pulse technique [8], the teleportation experiment consisted of four operations: (i) an entangling 2-qubit gate, (ii) a 2-qubit mapping of the Bell basis to the computational basis, (iii) decoherence of two qubits in the computational basis, and (iv) a conditional 3-qubit unitary. Our model incorporates the decoherence step as a mixture of classical rotations of the hidden spins. Since the model can account for $K=3$ entangling gates without loss of signal, it provides a gate-by-gate classical model whose predictions are identical to the quantum predictions. This experiment therefore does not demonstrate genuine quantum-information processing in the sense of our third criterion. For a larger value of $\alpha$, when $K=2$, our model predicts a drop in signal by a factor of $\eta=1 / 33$ at the third entangling step. Nevertheless, even when $K=2$, the classical model can still account for the teleportation experiment if the first two 2-qubit gates are "compiled" into a single 3-qubit gate.

Similar conclusions apply to the other 3-qubit experiments performed to date [5-8] and to most of the 2-qubit experiments [11 14,8, 15, 16]. The 2-qubit experiment described in [9] ran through 15 entangling gates with an approximately exponential signal-to-noise decrease that is, however, much slower than the factor of $\eta=1 / 9$ per entangling gate predicted by our model after the first $K=5$ steps. The 2-qubit experiment reported in [10] implemented up to 14 entangling gates, but the absence of signal-to-noise data makes comparison with our model difficult. 
This paper, together with [23], begins the task of establishing standards for assessing the quantumness of bulk-ensemble NMR quantum computation 33,34. Our gate-by-gate classical model erects a hurdle in the way of NMR quantum-information processing. "Testing" our classical model is not the point, for no one would contend that it describes the physics that underlies an NMR experiment. The point is that experiments that fail to clear the hurdle can be explained in classical language and thus do no genuine quantum-information processing according to our third criterion.

It will be easy for NMR experimenters to clear our hurdle, by measuring the signal-tonoise ratio in experiments involving many entangling gates, like the experiment reported in [9]. Yet jumping over our hurdle is probably not sufficient to provide a warranty of genuine quantum-information processing, for that would require showing that a given experiment is inconsistent with all gate-by-gate classical models, not just the model formulated in this paper. There are reasons for believing that our classical model is not optimal, the most cogent of which is that the model's transition probabilities for an entangling gate transform qubits that are unaffected by the gate. Avenues for improving the model include the following: (i) investigating more efficient representations both for quantum states near the maximally mixed density operator and for the transition probabilities between states, (ii) seeking gate representations that are more efficient when fewer qubits are involved in the gate, and (iii) addressing the extent to which one is allowed to "compile" successive entangling unitaries into a single operation (compiling is routine in NMR experiments as a method for reducing the length of a computation).

We conjecture, however, that no matter how efficient the gate representations are made, an ultimate signal decrease is an unavoidable consequence of any attempt to describe entangling unitaries classically, even when the unitaries act only on separable states. Indeed, more interesting than our results would be a demonstration of this conjecture. Should the conjecture prove to be correct, one could conclude that the quantumness of NMR quantum computation, for small numbers of qubits, lies in the ability to avoid any signal decrease. More broadly, we speculate that the power of quantum-information processing comes not from entanglement itself, but rather from the information-processing capabilities of entangling unitaries 26.

\section{ACKNOWLEDGMENTS}

Thanks to S. L. Braunstein for drawing our attention to the question of the quantumness of NMR quantum computation, to R. Jozsa, M. Grassl, and D. Janzing for useful comments, and to C. A. Fuchs for a careful reading of and suggestions for improving the manuscript. This work was supported in part by the US Office of Naval Research (Grant No. N0001493-0-0116) and by the UK Engineering and Physical Sciences Research Council. 


\section{REFERENCES}

[1] D. G. Cory, A. F. Fahmy, and T. F. Havel, Proc. Nat. Acad. Sci. USA 94, 1634 (1997).

[2] N. A. Gershenfeld and I. L. Chuang, Science 275, 350 (1997).

[3] W. S. Warren, Science 277, 1688 (1997).

[4] M. A. Nielsen, E. Knill, and R. Laflamme, Nature 396, 52 (1998).

[5] D. G. Cory et al., Phys. Rev. Lett. 81, 2152 (1998).

[6] R. Laflamme, E. Knill, W. H. Zurek, P. Catasti, and S. V. S. Mariappan, Phil. Trans. Roy. Soc. London A 356, 1941 (1998).

[7] N. Linden, H. Barjat, and R. Freeman, Chem. Phys. Lett. 296, 61 (1998).

[8] D. G. Cory, M. D. Price, and T. F. Havel, Physica D 120, 82 (1998).

[9] J. A. Jones and M. Mosca, unpublished, e-print quant-ph/9808056.

[10] I. L. Chuang, N. Gershenfeld, and M. G. Kubinec, Phys. Rev. Lett. 80, 3408 (1998).

[11] I. L. Chuang, N. Gershenfeld, M. G. Kubinec, and D. W. Leung, Proc. Roy. Soc. London A 454, 447 (1998).

[12] I. L. Chuang, L. M. K. Vandersypen, X. Zhou, D. W. Leung, and S. Lloyd, Nature 393, 143 (1998).

[13] E. Knill, I. Chuang, and R. Laflamme, Phys. Rev. A 57, 3348 (1998).

[14] D. G. Cory, A. E. Dunlop, T. F. Havel, S. S. Somaroo, and W. Zhang, unpublished, e-print quant-ph/9809045.

[15] J. A. Jones, M. Mosca, and R. H. Hansen, Nature 393, 344 (1998).

[16] J. A. Jones and M. Mosca, J. Chem. Phys. 109, 1648 (1998).

[17] J. A. Jones, R. H. Hansen, and M. Mosca, J. Magn. Resonance 135, 353 (1998).

[18] G. P. Berman, G. D. Doolen, G. V. López, and V. I. Tsifrinovich, Phys. Rev. B 58, 11570 (1998).

[19] E. Knill and R. Laflamme, Phys. Rev. Lett. 81, 5672 (1998).

[20] L. J. Schulman and U. Vazirani, unpublished, e-print quant-ph/9804060.

[21] B. E. Kane, Nature 393, 133 (1998).

[22] D. P. DiVincenzo, review of [30] in Quick Reviews in Quantum Computation and Information, http://quickreviews.org

[23] S. L. Braunstein et al., unpublished, e-print quant-ph/9811018.

[24] That entanglement is the "essential non-classical feature providing the computational speed-up in the known quantum algorithms" is argued forcefully by A. Ekert and R. Jozsa, Phil. Trans. Roy. Soc. London A 356, 1769 (1998).

[25] W. H. Zurek, in replying [6] to a question about whether several image-processing techniques can be regarded as quantum-information processing, expresses the general view: "None of the examples invoked make an explicit use of arbitrary quantum superpositions or entanglement for information processing. Thus, while of interest, they cannot be regarded as 'quantum computation' ... ."

[26] Knill and Laflamme [19] consider a model for ensemble computation using input states in which one qubit is in a pure state and all the other qubits are in a maximally mixed state. They note that although the apparent power of quantum computers "is frequently attributed to 'quantum parallelism,' interference phenomena derived from the superposition principle, and the ability to prepare and control pure states according to the Schrödinger equation," their results suggest that "the usual reasons given for why quantum computation appears to be so powerful may have to be revised." 
[27] It is likely that there are "nonlocal" hidden-variable models for $N$ qubits which reproduce all the predictions of quantum mechanics, but which violate the assumptions of our third criterion, in that the transition probabilities for a gate would depend on the initial state or would include qubits not involved in a gate in a nontrivial way.

[28] Atta-ur-Rahman and M. I. Choudhary, Solving Problems with NMR Spectroscopy (Academic Press, San Diego, 1996).

[29] G. Vidal and R. Tarrach, Phys. Rev. A 59, 141 (1999).

[30] K. Życzkowski, P. Horodecki, A. Sanpera, and M. Lewenstein, Phys. Rev. A 58, 883 (1998).

[31] For a complete discussion, see [29 and references cited therein on measures of entanglement.

[32] L. J. Landau and R. F. Streater, Linear Algebra and Its Applications 193, 107 (1993).

[33] Although [20] shows that bulk-ensemble NMR is scalable to many qubits, the proper scaling sets in at such a large number of qubits, $N \sim \alpha^{-2} \simeq 10^{9}-10^{11}$, that it is irrelevant to the present paper.

[34] Some authors have been careful about advancing claims for the quantum nature of NMR quantum-information processing. For example, Cory et al., in a paper [8] outlining the principles of NMR quantum computation, comment (in the abstract) that "nuclear magnetic resonance spectroscopy is capable of emulating (our emphasis) many of the capabilities of quantum computers, including unitary evolution and coherent superpositions, but without attendant wave-function collapse." Others have been less cautious. Gershenfeld and Chuang, in their original paper on NMR [2], remark, "Because the $N$ spins in each molecule may be in entangled quantum superposition states, our computer can be a quantum one," and Chuang et al. [11], in describing the experiment referred to at the end of Sec. III, which implements a single 2-qubit gate (a controlled-NOT), note, "Perhaps the most interesting aspect of this experiment is that the experimental results cannot be explained by a classical model of two interacting spins (of spin $\frac{1}{2}$ )." 